\title{
Perfil da qualidade de vida de idosos com hipertensão arterial em uma ESF no
}

\section{interior do Mato Grosso}

\author{
Quality of life profille of elderly people with hypertension in an ESF in the interior of Mato Grosso \\ Perfil de calidad de vida de ancianos con hipertensión en una ESF del interior de Mato Grosso
}

Recebido: 01/10/2021 | Revisado: 17/10/2021 | Aceito: 10/11/2021 | Publicado: 15/11/2021

\author{
Rosana Maria de Moraes \\ ORCID: https://orcid.org/0000-0002-3019-0277 \\ Agencia Educacional Brasileira, Brasil \\ E-mail: rosana38silva@hotmail.com
}

\begin{abstract}
Resumo
Objetivou-se com esse estudo, avaliar a qualidade de vida de idosos com hipertensão arterial da ESF- São Benedito município de Poconé - MT. Este trabalho consiste em um estudo epidemiológico, transversal, quantitativo de abordagem descritiva, através de dados secundários, referente aos hipertensos diagnosticados e cadastrados na ESF. Os resultados encontrados, foram organizados por meio da análise de sessenta questionários, com os dados dos idosos cadastrados, onde demostraram que $89,4 \%$ declararam ser casados $11,6 \%$ declararam ser solteiros. Em que pese à condição filhos, $98 \%$ declararam ter filhos. Verificou-se que o maior percentual de idosos, 67,6\%, afirmaram ser o chefe da família, seguido de $32,9 \%$ que admitiram ser o esposo ou outros. Já os dados em relação ao perfil de hábitos e saúde, os resultados apontaram que 79,4 \% moram na zona urbana e esses não fazem uso de bebida alcoólica e apenas 16, 7\% são tabagistas. Em relação à associação com tabagismo o estudo encontrou aproximação com a literatura, pois $16,7 \%$ dos pacientes apresentam associação com tabagismo. Os resultados da prática de atividade física, do presente estudo $36 \%$ realiza algum tipo de atividade com frequência predominante e em $50 \%$ da população de três vezes ou mais por semana. Quanto aos hábitos de lazer 100,0 \% dos avaliados afirmaram que realizam algum tipo de atividade física. Considera-se que os propósitos estabelecidos para este estudo foram alcançados visto que foi possível conhecer parte da realidade dos idosos cadastrados pelo Programa HiperDia com ênfase na qualidade de vida dos mesmos.

Palavras-chave: Hipertensão arterial; Promoção da saúde; Qualidade de vida.
\end{abstract}

\begin{abstract}
The objective of this study was to evaluate the quality of life of elderly people with arterial hypertension in the ESFSão Benedito municipality of Poconé - MT. This work consists of an epidemiological, cross-sectional, quantitative study with a descriptive approach, through secondary data, referring to diagnosed hypertensive patients registered in the FHS. The results found were organized through the analysis of sixty questionnaires, with data from the registered elderly, which showed that $89.4 \%$ declared to be married $11.6 \%$ declared to be single. Regarding the condition of children, $98 \%$ declared having children. It was found that the highest percentage of elderly people, $67.6 \%$, claimed to be the head of the family, followed by $32.9 \%$ who admitted to being the spouse or others. As for the data regarding the profile of habits and health, the results showed that $79.4 \%$ live in urban areas and these do not use alcoholic beverages and only $16.7 \%$ are smokers. Regarding the association with smoking, the study found similarity with the literature, as $16.7 \%$ of patients are associated with smoking. The results of the practice of physical activity, in this study, $36 \%$ perform some type of activity with predominant frequency and in $50 \%$ of the population three times or more a week. As for leisure habits, $100.0 \%$ of those evaluated stated that they perform some type of physical activity. It is considered that the purposes established for this study were achieved since it was possible to know part of the reality of the elderly registered by the HiperDia Program with an emphasis on their quality of life.
\end{abstract}

Keywords: Hypertension; Health promotion; Quality of life.

\section{Resumen}

El objetivo de este estudio fue evaluar la calidad de vida de los ancianos con hipertensión arterial en el municipio de Poconé - MT de la ESF-São Benedito. Este trabajo consiste en un estudio epidemiológico, transversal, cuantitativo con enfoque descriptivo, a través de datos secundarios, referido a pacientes hipertensos diagnosticados registrados en la ESF. Los resultados encontrados se organizaron a través del análisis de sesenta cuestionarios, con datos de los ancianos registrados, que arrojaron que el 89,4\% declaró estar casado y el 11,6\% declaró ser soltero. En cuanto a la condición de los hijos, el 98\% declaró tener hijos. Se encontró que el mayor porcentaje de personas mayores, el 67,6\%, afirmó ser el jefe de familia, seguido del 32,9\% que admitió ser cónyuge u otras personas. En cuanto a los datos sobre el perfil de hábitos y salud, los resultados arrojaron que el 79,4\% vive en zonas urbanas y estas no consumen bebidas alcohólicas y solo el $16,7 \%$ son fumadores. En cuanto a la asociación con el tabaquismo, el estudio encontró similitud con la 
literatura, ya que el 16,7\% de los pacientes están asociados con el tabaquismo. Los resultados de la práctica de actividad física, en este estudio, el 36\% realiza algún tipo de actividad con frecuencia predominante y en el 50\% de la población tres o más veces por semana. En cuanto a los hábitos de ocio, el 100,0\% de los evaluados afirma realizar algún tipo de actividad física. Se considera que los propósitos establecidos para este estudio se cumplieron ya que se pudo conocer parte de la realidad de los adultos mayores registrados por el Programa HiperDia con énfasis en su calidad de vida.

Palabras clave: Hipertensión; Promoción de la salud; Calidad de vida.

\section{Introdução}

De acordo com a Organização Mundial da Saúde (OMS), cerca de 7 milhões de pessoas morrem a cada ano e 1,5 bilhão adoecem por causa da hipertensão arterial sistêmica (HAS). As graves consequências da doença podem ser evitadas, desde que os hipertensos conheçam sua condição e mantenham-se em tratamento (SOCIEDADE BRASILEIRA DE HIPERTENSÃO, 2016).

O envelhecimento é um processo dinâmico e progressivo que causa diversas alterações no organismo, sejam elas de ordem morfológica, psicológica, funcional ou biológica, levando à diminuição da capacidade funcional e ao desenvolvimento de doenças crônicas não transmissíveis. Dentre os fatores de risco para o desenvolvimento das doenças crônicas não transmissíveis, a hipertensão arterial sistêmica é o mais prevalente (Boing, 2007).

A HAS é definida como a manutenção de níveis de pressão arterial acima de $140 \mathrm{mmHg}$ na sistólica e $90 \mathrm{mmHg}$ na diastólica. Estão relacionadas a fatores intrínsecos, como hereditariedade, sexo, idade e raça, e a fatores extrínsecos, como tabagismo, sedentarismo, obesidade, estresse, dislipidemia e dieta. Além disso, há aumento do risco de comorbidades, como infarto agudo do miocárdio, acidente vascular encefálico e insuficiência renal crônica (Cesarino et al., 2008).

Ainda assim, doenças e limitações não são resultados inevitáveis do envelhecimento, existem grandes evidências de que alterações próprias do envelhecimento tornam o indivíduo mais disposto ao surgimento de HAS, sendo esta a principal doença crônica na população idosa (Veras, 2009).

De acordo com Lipp (2007) não praticar atividade física tem-se tornado como um fator determinante para a ocorrência de mortes e doenças. Estudos em todo o país identificou que a longo prazo a realização de atividade física regular possui efeito protetor ou preventivo para as doenças crônicas.

Em outro estudo foi analisado que as atividades desgastantes no ambiente de trabalho também podem gerar danos à saúde. Entre elas estão as alterações cardiovasculares e hipertensão arterial. Em um estudo realizado com profissionais da saúde identificou-se que as longas jornadas de trabalho, pressão do tempo, altas demandas, são fatores associados a transtornos mentais, estresse, e a hipertensão arterial (Albuquerque; Lira; Lopes, 2010).

Apesar das dificuldades na adesão ao tratamento, os avanços no conhecimento e a evolução obtida na terapêutica têm aumentado à expectativa de vida da população. Com a longevidade, é importante que os indivíduos mantenham a autonomia e a saúde, pois o envelhecimento aumenta o risco de doenças crônicas, sobretudo as cardiovasculares (Brandão et al., 2013).

Além disso, com o aumento da sobrevida dos pacientes com doenças crônicas ou graves, a qualidade de vida passou a ser mais valorizada e a importância de sua avaliação foi reconhecida e incorporada aos ensaios clínicos. As informações sobre qualidade de vida dos hipertensos, em estudos nacionais, ainda são escassas, e dados comparativos com a população geral são importantes dentro desse contexto (Pereira et al., 2008).

Justifica-se esse estudo, tendo em vista a necessidade de conhecer o perfil de idosos portadores de hipertensão arterial e promover a promoção de saúde bem como orientar sobre uma melhor qualidade de vida, e desta forma influenciar na evolução ou melhora das mesmas. Portanto a partir desse contexto, é fundamental estudar o perfil de saúde de idosos diagnosticados com hipertensão arterial, visto que tal doença está associada a fatores importantes e controláveis. Assim objetivou-se com esse estudo, avaliar a qualidade de vida de idosos com hipertensão arterial da ESF- São Benedito município de Poconé - MT. 


\section{Metodologia}

Este trabalho consiste em um estudo epidemiológico, transversal, quantitativo de abordagem descritiva, através de dados secundários (Gil, 2008), referente aos hipertensos diagnosticados e cadastrados, em uma ESF especifica em Poconé - MT.

O estudo foi realizado na ESF-São Benedito no município de Poconé, na zona urbana da cidade, a unidade fica próxima ao centro da cidade, o lugar atende toda a região do bairro onde a unidade está inserida. A população do estudo contava com 60 idosos cadastrado no programa Hiperdia daquela unidade, onde o grupo de estudo apresentava características distintas.

O instrumento que foi utilizado para realização desse estudo foi um questionário semi-estruturado elaborado pela própria autora com as seguintes perguntas: Nome, idade, raça/cor, sexo, peso, zona, local de nascimento, data do diagnóstico e início do tratamento. Após a coletada desses dados geraram o resultado do estudo.

\section{Resultados e Discussão}

Os resultados encontrados, foram organizados por meio da análise de sessenta questionários, com os dados dos idosos cadastrados. Assim os resultados demostraram que 89,4\% declararam ser casados 11,6\% declararam ser solteiros. Em que pese à condição filhos, $98 \%$ declararam ter filhos. Verificou-se que o maior percentual de idosos, 67,6 \%, afirmaram ser o chefe da família, seguido de $32,9 \%$ que admitiram ser o esposo ou outros.

O envolvimento do cônjuge é componente facilitador para a adesão ao tratamento, contribuindo desta forma para o controle da PA. Em um estudo com 200 hipertensos realizado em Maceió - AL, 98 \% dos hipertensos casados apresentavam PA controlada e, entre os solteiros, a frequência de controle correspondeu a $79 \%$ (Freitas et al., 2012).

A maioria dos pacientes estudados 89,4\% eram casados, da mesma forma que nos estudos de Lopes et al. (2012), Pierin et al. (2001), Cenatti et al, (2013) e Zaitune et al. (2006) também houve um predomínio de pessoas que vivem com cônjuge. Esta condição pode estar associada a um acúmulo de funções domésticas e obrigações, ou a certo estresse proporcionado pela constituição de família assim configurando um dos fatores de risco.

Já os dados em relação ao perfil de hábitos e saúde, os resultados apontaram que 79,4 \% moram na zona urbana e esses não fazem uso de bebida alcoólica e apenas 16, 7\% são tabagistas. A baixa prevalência do consumo de bebida alcoólica no grupo estudado pode ser considerada como um fator positivo nesta população em que 79,4 \% do grupo estudado não fazem uso de bebida alcoólica, visto que moram na zona urbana.

O consumo elevado de álcool associa-se à maior mortalidade total, morte súbita arrítmica, hipertensão arterial, cardiomiopatia, acidente vascular cerebral hemorrágico, doença hepática e pancreática, e diversas formas de câncer. Uma das dificuldades de ter o controle do uso de bebidas alcoólicas é o fato de existir a aceitação social da bebida. A literatura aponta que os prejuízos apontados pelas pessoas à bebida alcoólica estão mais associados à forma e a frequência diária ou semanal como ela é usada, do que ao seu uso em si (Lima, 2004).

Em relação à associação com tabagismo o estudo encontrou aproximação com a literatura, pois 16,7 \% dos pacientes apresentam associação com tabagismo. Estudos realizados em Campinas evidenciaram que entre pacientes hipertensos com mais de sessenta anos, 11,9 \% eram tabagistas e estudos realizado no Sul evidenciou que entre pessoas cadastradas em um Grupo Hiperdia 15,7 \% eram tabagistas e destes a população masculina apresentava maior propensão ao tabagismo que a feminina (Zaitune et al., 2006; Foletto, 2009).

Referente os resultados da prática de atividade física, do presente estudo $36 \%$ realiza algum tipo de atividade com frequência predominante e em $50 \%$ da população de três vezes ou mais por semana. Quanto aos hábitos de lazer 100,0 \% dos avaliados afirmou positivamente para esse item de acordo com a Figura 1. 
Figura 1 - Dados sobre quanto aos hábitos de lazer pelos idosos cadastrados em Poconé -MT, 2019.

\section{Hábitos de Lazer}

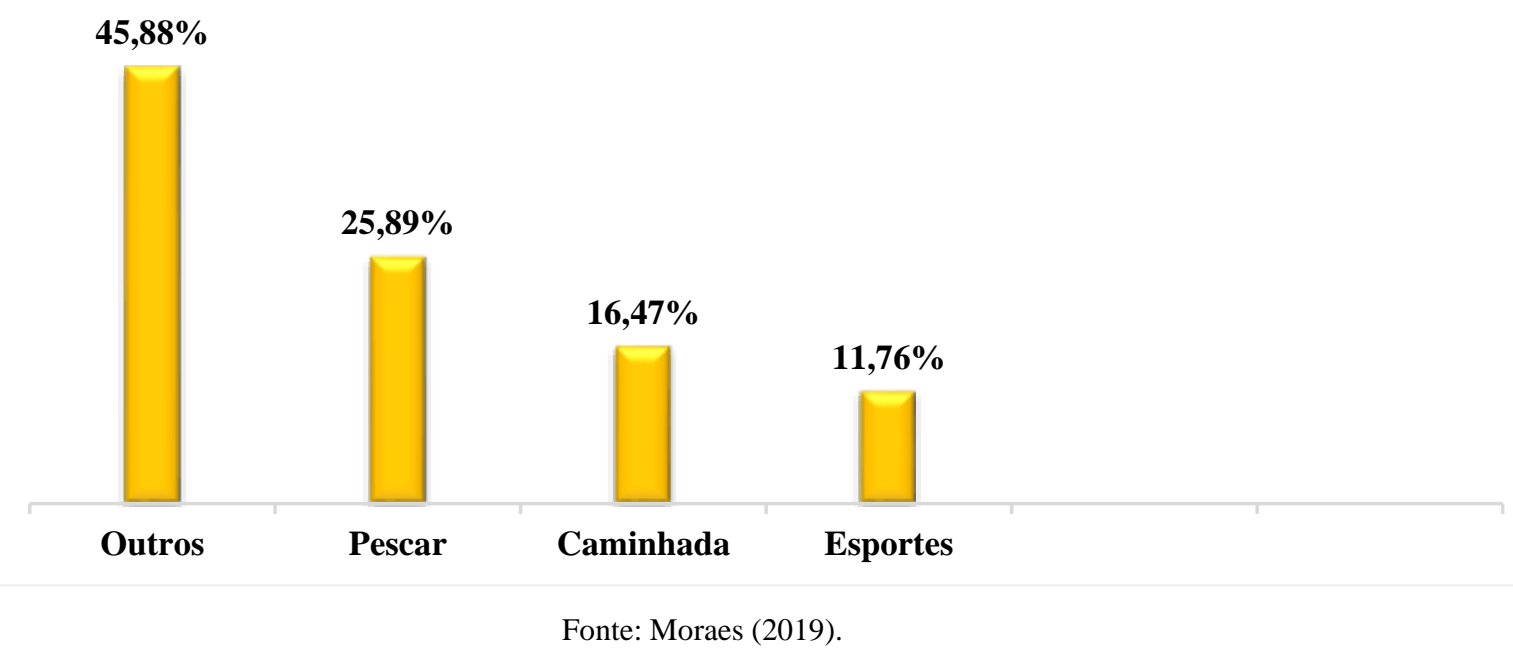

Esses resultados mostram que os idosos estão buscando por diversos caminhos para alcançar a qualidade de vida.

A realização de atividade regular auxilia na redução de risco coronário, aumenta os níveis de lipoproteína de alta densidade, diminui os níveis de triglicérides, reduz a pressão arterial, auxilia na redução de poso corporal, melhora a tolerância à glicose e corrige a distribuição da gordura corporal. Um benefício importante de se realizar atividade física em idosos com osteoporose é conservação da densidade óssea, além de contribuir para a flexibilidade e o aumento da força muscular. A atividade física melhora a autoestima e, consequentemente, a qualidade de vida, sendo assim reduz a morbidade (Gravina, 2007).

Além do mais, a atividade física contribui para a redução e controle do peso, diminuindo o risco de complicações decorrente da HAS e DM promovendo interação social entre idosos, preserva a independência nas atividades da vida diária.

A pratica da atividade, quando ocorre de forma regular e moderado são igualmente essenciais para o tratamento da HAS e da obesidade e são fundamentais na vida diária. Equilibra o excesso de consumo calórico, baixa os níveis do colesterol, melhora os níveis da glicemia no sangue e promove o relaxamento e a diminuição de estresse, fator igualmente significativo nesta problemática (Mendes, 2008).

Daí a importância de serem incorporados hábitos de vida saudáveis como a atividade física, associados aos medicamentos, a fim de reduzir a quantidade ministrada no mesmo. O elevado nível de atividade física diária correlaciona-se com menores níveis de pressão arterial em repouso. Além disso, o exercício físico aeróbio reduz cerca de 3,8 mmHg da pressão sistólica e $2 \mathrm{mmHg}$ da pressão diastólica (Guimarães, 2004).

Para Pacheco et al. (2009) a qualidade de vida da população idosa traz informações que podem ser utilizadas para avaliar a eficácia de alguns tratamentos de doenças crônicas como a hipertensão. Os agravos à saúde e o impacto físico e psicossocial na vida dos indivíduos, podem ser mensuradas através de certos instrumentos, oferecendo aos mesmos a oportunidade de modificar seus hábitos de vida através da percepção do seu próprio estado geral de saúde.

A promoção da saúde, mais do que um conceito, tem sido considerada um modelo, um modo de pensar e de operar articulado as demais políticas e tecnologias desenvolvidas no sistema de saúde brasileiro, que vai contribuir na construção de ações que possibilitam responder ás necessidades sociais em saúde da população (BRASIL, 2006).

Isso presume uma concepção de saúde que não está restrita a ausência de doença, mas que seja capaz de atuar sobre seus determinantes, o que incide nas condições de vida da população. Está além da prestação de serviço clínico-assistenciais, 
supondo ações inter-setoriais que envolvam a educação, o saneamento básico, a moradia, o trabalho e a renda, a alimentação, o meio ambiente, o acesso a bens e serviços essenciais e também ao lazer entre outros determinantes sociais da saúde (Sícoli, 2003).

A promoção da saúde estreita então sua relação com a vigilância em saúde, numa articulação que reforça a exigência de um movimento integrador na construção de consensos e sinergias e na execução de agendas governamentais a fim de que políticas públicas sejam cada vez mais favoráveis à saúde e à vida, e estimulem e fortaleçam o protagonismo dos cidadãos em sua elaboração e implementação, ratificando os preceitos constitucionais da participação social (BRASIL, 2006).

Para Pelicioni e Pelicioni (2007) a comunidade deve ter o direito e a responsabilidade de tomar decisões que sejam viáveis e que se conjugue a favor da vida de seus membros e para isso tem que ser preparada, pois os indivíduos só aprendem a participar, participando e a participação depende de uma educação democrática e libertadora.

As autoras supracitadas, observam que estimular o interesse por cuidar de sua própria saúde e ajudar a população a reconhecer os fatores que a torna vulnerável no processo saúde e adoecimento faz parte da responsabilidade educativa. Para isso, a educação em saúde deve ser crítica, problematizadora da realidade compartilhada e reflexiva como um processo intencional como o objetivo de prover situações ou experiências que estimulem a expressão potencial dos seres humanos (Pelicioni \& Pelicioni, 2007).

Assim, os profissionais de saúde atuantes em saúde da família e em áreas afins, reconhece que é de extrema importância conhecer o perfil dos usuários hipertensos cadastrados e acompanhados na ESF, tendo em vista o planejamento de ações em saúde que possam contribuir para a melhoria do atendimento e da qualidade de vida desse grupo de usuários (Santos et al., 2013).

\section{Conclusão}

Portanto, a hipertensão arterial é apontada como fator de risco para complicações e doenças cardiovasculares na sociedade atual, tais como morte súbita, edema agudo de pulmão, insuficiência renal, infarto agudo do miocárdio e acidente vascular encefálico, explicando $54 \%$ das mortes por acidente vascular encefálico e $47 \%$ daquelas por doença isquêmica do coração. Considera-se que os objetivos estabelecidos para este estudo foram alcançados visto que foi possível conhecer parte da realidade dos idosos cadastrados pelo Programa HiperDia com ênfase na qualidade de vida dos mesmos.

Portanto a hipertensão é mais prevalente em determinados subgrupos da população como os idosos do sexo masculino. Ainda que as políticas públicas devam contemplar a todos, deve ter a atenção especial voltada para os subgrupos mais vulneráveis, tanto para as ações de prevenção, de controle da hipertensão, assim como para as de promoção à saúde.

Assim, com este estudo foi possível constatar que a hipertensão arterial, é o principal fator de risco de morte entre as doenças não transmissíveis, mostra relação direta com o risco cardiovascular. Entretanto, apesar dos progressos na prevenção, no diagnóstico, no tratamento e no controle, ainda é importante problema de saúde pública.

Devem ser metas dos profissionais de saúde a identificação precoce e a abordagem adequada dos fatores de risco para o desenvolvimento da hipertensão arterial, principalmente na população de alto risco.

Entre as medidas preventivas, destacam-se a adoção de hábitos alimentares saudáveis, a prática de atividade física e o abandono do tabagismo. Em relação às ações do enfermeiro relacionadas à promoção à saúde e prevenção da hipertensão arterial é imprescindível a sua atuação integralizada e interdisciplinar por ser um educador em saúde, através do desenvolvimento de estratégias de educação, objetivando a conscientização dos indivíduos e comunidade sobre a busca e manutenção da saúde.

Ressalta-se ainda, a importância das ações de enfermagem realizadas pelo enfermeiro em relação à hipertensão arterial, pois conseguem demonstrar de forma clara a real necessidade de prevenção e promoção da saúde determinada pela Constituição, somente a prevenção, o controle e a eficácia na detecção da doença poderão diminuir a possibilidade de outras complicações. 
Assim, pode-se organizar, planejar e traçar planos de ações a curto, médio e longos prazos com os profissionais de saúde, no intuito de suprir as necessidades de cada indivíduo, além de promover, proteger e recuperar a saúde destes. Pelo exposto, acredita-se que realizar o diagnóstico desses fatores não foi o único desafio, consideram-se também outros aspectos, como o de verificar o impacto desses fatores na qualidade de vida dos pacientes. Percebe-se a necessidade de ampliar ainda mais este estudo, para se puder ter um diagnóstico situacional completo, contendo todos os níveis de complexidade existente destes usuários.

\section{Referências}

Albuquerque, J. G., Lira, A. L. B. C. \& Lopes, M. V. O. (2010). Fatores preditivos de diagnósticos de enfermagem em pacientes submetidos ao transplante renal. Rev. Bras. Enferm, Brasília, 63(1), 98-103.

Boing, A. C. \& Boing, A. F. (2007). Hipertensão arterial sistêmica: o que nos dizem os sistemas brasileiros de cadastramentos e informações em saúde. Revista Brasileira de Hipertensão.

Brandão, A. P., Brandão, A. A., Magalhães, M. E. C. \& Pozzan, R. (2003). Epidemiologia da hipertensão arterial. Revista de Cardiologia. Estado de São Paulo. BRASIL. (2006). Ministério da saúde. Secretaria de Vigilância em Saúde, Secretaria de Atenção á Saúde. Política Nacional de Promoção da Saúde. Brasília, DF, 60 .

BRASIL. Ministério da Saúde (MS). (2013). Secretaria de Atenção à Saúde. Departamento de Atenção Básica. Política Nacional de Alimentação e Nutrição. Brasília: MS.

Cenatti, J. L., Lentsck, M. H., Prezotto, K. H. \& Pilger, C. (2013). Caracterização de usuários hipertensos de uma unidade básica de saúde da família. Revista de Enfermagem e Atenção à Saúde, Paraná, 2(1), 21-31.

Cesarino, C. B., Cipulo, J. P., Martin, J. F. V., Ciorlia, L. A., Godoy, M. R. P., Cordeiro, J. A. \& Rodrigues, I. C. (2008). Prevalência e fatores sociodemográfico em hipertensos de São José do Rio Preto - SP. Arq Bras Cardiologia, 9(1), 31 - 35.

Foletto, K. C. (2009). Perfil epidemiológico, estado nutricional e fatores associados à hipertensão e diabetes mellitus em idosos cadastrados na HiperDia do município de Caixias do Sul (RS). Dissertação (Pós-graduação latu senso em saúde pública) - faculdade de Medicina, Universidade federal do Rio Grande do Sul, 46.

Freitas, L. C., Rodrigues, G. M., Araújo, F. C., Falcon, E. B. S., Xavier, N. F., Lemos, E. L. C. \& Pires, C. A. A. (2012). Perfil dos hipertensos da Unidade de Saúde da Família Cidade Nova 8, município de Ananindeua-PA. Rev bras med fam comunidade. Florianópolis.

GIL, A. C. (2008). Métodos e técnicas de pesquisa social. 6a. ed. São Paulo: Atlas.

Gravina, C. F., Grespan, S. M. \& Borges, J. L. Tratamento não-medicamentoso da hipertensão no idoso. Rev. Bras. Enfermagem, 14(1), 33-36.

Guimarães, G. V. \& Ciola, C. E. G. (2004). Síndrome Metabólica: Abordagem do Educador Físico. Rev. Soc. Cardiologia.

Lima, M. T., Bucher, J. S. N. F. \& Lima, J. W. O. (2004). A Hipertensão Arterial sob o olhar de uma população carente: Estudo exploratório a partir dos conhecimentos, atitudes e práticas. Cad. Saúde Pública.

Lipp, M. E. N. (2007). Controle do estresse e hipertensão arterial sistêmica. Rev. Bras. Hipertensos. Campinas, 14(2), 89- 93.

Lopes, W. S., Guedes, T. A., Araújo, S. M. A. \& Gomes, A. M. L. Arterial hypertension: sociodemographic profile and comorbidities of patients from northwest Paraná State, Southern Brazil. Acta Scientiarum. Health Sciences, Maringá, 34(2), 119-126.

Mendes, R. \& Barata, J. L. T. (2008). Exercício aeróbio e pressão arterial no idoso. Rev Portal Clinica Geral. v. 24, p. $251-257$.

Pacheco, L. R., Lima, M. C. C., Nogueira, A. L. G. \& Barbosa, M. A. (2009). A avaliação de qualidade de vida: guia para profissionais de saúde.

Pelicioni, M. C. F. \& Pelicioni, A. F. (2007). Educação e promoção de saúde: uma retrospectiva histórica. Mundo da Saúde, São Paulo, $31(3), 320-328$.

Pereira, J. C., Barreto, S. M. \& Passos, V. M. (2008). O perfil de saúde dos idosos brasileiros precisa melhorar: estudo de base populacional. Arq. Bras. Cardiologia.

Pierin, A. M. G., Mion Junior, D., Fukushima, J. T., Pinto, A. R. \& Kaminaga, M. M. O perfil de um grupo de pessoas hipertensas de acordo com conhecimento e gravidade da doença. Revista da Escola de Enfermagem da USP, 35(1), 11-18.

Santos, A. B. V., Barreto, V. P., Oliveira, S. M., Gomes, C. A. M., Viana, K. M., Brasil, B. O. \& Abrantes, T. G. (2013). Perfil Epidemiológico da Hipertensão Arterial Sistêmica na população de Cajazeiras, Paraíba. Revista Brasileira de Ciências da Saúde, 17(3), $253-262$.

Sícoli, J. L. \& Nascimento, P. R. (2003). Promoção de saúde: conceito, princípios e operacionalização. Interface-Comunicação, Saúde, Educação, Botucatu, 7(12),91- 112 .

Sociedade brasileira de cardiologia. (2016). VII Diretrizes Brasileiras de Hipertensão. Arq. Bras. Cardiologia, 107(3). 
Research, Society and Development, v. 10, n. 15, e21101521326, 2021

(CC BY 4.0) | ISSN 2525-3409 | DOI: http://dx.doi.org/10.33448/rsd-v10i15.21326

Veras, R. (2009). Envelhecimento populacional contemporâneo: demandas, desafios e inovações. Rev. Saúde Pública.

Zaitune, M. P. A., Barros, M. B. A., César, C. L. G., Carandina, L. \& Goldbaum, M. (2006). Hipertensão arterial em idosos: prevalência, fatores associados e práticas de controle no município de Campinas, São Paulo, Brasil. Caderno de Saúde Pública, Campinas, 22(2), 285-294. 